\title{
Prediction of Road Traffic Accidents Based on Grey System Theory and Grey Markov Model
}

\author{
Xiaohua Jin ${ }^{1}$, Jiyu Zheng ${ }^{2 *}$, Xingyue Geng ${ }^{2}$ \\ ${ }^{1}$ School of Energy \& Environment Engineering, Zhongyuan University of Technology, Zhengzhou 450007, China \\ ${ }^{2}$ School of Safety Engineering, Henan University of Engineering, Zhengzhou 451191, China
}

Corresponding Author Email: zhengjiyu@ haue.edu.cn

https://doi.org/10.18280/ijsse.100214

Received: 12 December 2019

Accepted: 8 February 2020

\section{Keywords:}

road traffic accidents, grey system theory, grey

Markov model, prediction

\begin{abstract}
The economic boom in China has pushed up the car ownership, which in turn leads to a rise in road traffic accidents. Thus, it is important to predict the trend of road traffic accidents from the existing data on such accidents. This paper sums up the features of road traffic accidents in China in recent years, and predicts such accidents separately based on grey system theory and grey Markov model. The prediction results of the two methods were compared. Then, the influencing factors of road traffic accidents were identified, and several countermeasures were put forward to ensure traffic safety. The results show that the number of road traffic accidents, as well as the number of deaths, the number of injuries, and property loss caused by such accidents in China were falling before 2015, but all rebounded after 2015; the relative errors of grey prediction maximized at $7.24 \%$ and minimized at $0.49 \%$, while those of grey Markov model maximized at $1.96 \%$ and minimized at $0.02 \%$; the grey Markov model is obviously more accurate than grey prediction in the forecast of road traffic accidents. The research results provide a good reference for the prevention of road traffic accidents.
\end{abstract}

\section{INTRODUCTION}

Road traffic accidents cause different degrees of damage to various aspects, namely, public property, physical and mental health, and environment, posing a threat to the stable development of economy and life. This serious problem has attracted the attention from the government and the society. How to prevent road traffic accidents has long been a hot topic among experts at home and abroad [1-3]. For a long time, road traffic accidents have been viewed as a key difficulty in the research of traffic safety. Many domestic and foreign scholars have probed deep into traffic safety in multiple dimensions, and achieved fruitful results [4-6].

The world is a "grey" place where uncertainties abound. In 1982, Prof. Deng Julong proposed the grey system theory [7]: the uncertainties in large samples could be tackled by probabilistic and statistical methods, and uncertainty problems could be solved by fuzzy methods.

The grey system theory holds that any phenomenon, however complex it is, must have an overall function, and thus an inherent law [8]. The key is to find a proper way to mine and use the law. The regularity of any grey sequence could be revealed by weakening their stochasticity with a proper generation mechanism.

The prediction based on grey system theory is called grey prediction. The grey prediction of road traffic accidents is to fit the original data with an exponential curve. The geometry of the prediction result is a smooth curve. Hence, grey prediction performs poorly facing the highly-volatile data sequence of road traffic accidents [9-11]. This problem could be solved effectively by heuristics, Markov, and other strategies $[12,13]$.
The Markov model was created in 1906 by Russian mathematician Andrei Andreyevich Markov. The model involves some basic concepts and derived theories. Markov presented and examined the Markov chain, a general scheme for mathematical analysis of natural processes, and explored deep into Markov process, i.e. a stochastic process with no aftereffect.

The causes of road traffic accidents are sudden and stochastic. The probability of road traffic accident at the next moment has nothing to do with the situation at the current moment. These are exactly the features of the Markov chain. Therefore, the features of road traffic accidents could be investigated based on the Markov chain theory, laying the basis for traffic safety evaluation and decision-making [14-16].

The grey Markov model, a combination between grey prediction and Markov strategy, has been successfully applied in the prediction of road traffic accidents. For example, Li et al. [17], Hu [18], Gang [19] and Lin et al. [20] made reliable predictions of the number, causalities, and economic loss of road traffic accidents, using grey Markov model. The model also applies to forecasts of marine accidents, intelligent systems, operational status, and seasonal changes. For instance, Zhao and $\mathrm{Wu}$ [21] predicted marine accidents with grey Markov model. Lee et al. [22] predicted road traffic accidents accurately by combining grey Markov model and intelligent system theory. With the aid of grey Markov model, Liu et al. [23] predicted the bridge operation status, revealing the trend of bridge operation status in engineering. Chen and Zhu [24] analysed and predicted the seasonal changes of traffic accidents on expressways.

Considering the importance of traffic safety, this paper analyses the features of road traffic accidents of China in 
recent years, and then predicts such accidents by two separate methods: the basic grey model GM $(1,1)$ and grey Markov model. The prediction results of the two methods were compared. After that, the causes of road traffic accidents were identified, followed by the design of several countermeasures. Our research provides suitable prediction methods for road traffic accidents with different features, and promotes the accuracy and reliability of the predictions [25, 26]. The Markov model predicts the state of the object at the next moment according to the current state and the state transition probability matrix [27-29]. Therefore, state division is the basis of Markov prediction. The accuracy of Markov prediction relies on the appropriate division of states [30-33].

The remainder of this paper is organized as follows: Section 2 sums up the features of road traffic accidents; Section 3 predicts road traffic accidents through grey prediction; Section 4 forecasts road traffic accidents by grey Markov model; Section 5 compares the predicted results of the two methods; Section 6 identifies the causes and designs the countermeasures of road traffic accidents; Section 7 puts forward the conclusions.

\section{FEATURES OF ROAD TRAFFIC ACCIDENTS}

Car ownership is growing rapidly with the increase in resident income. Due to the rising number of vehicles, many roads are rather busy, with frequent occurrence of traffic jams. As a result, road traffic accidents have become commonplace.

Besides motor vehicles, roads are used by non-motor vehicles and pedestrians. Thus, road traffic accidents may occur between motor vehicles, between motor vehicles and non-motor vehicles, and between motor vehicles and pedestrians. With the surging number of electric bicycles, the accidents between motor vehicles and electric bicycles also rockets up.

Road traffic accidents are the main type of fatal accidents in most countries. The statistics of road traffic accidents mainly cover four aspects: the number of accidents, the number of deaths, the number of injuries, and property loss. In this paper, the data on the four aspects are collected from China Statistical Yearbooks.

As shown in Figure 1, the number of road traffic accidents in China decreased from 2010 to 2017, with a rebound in 2016. Due to the growth in car ownership and car travel, the number of road traffic accidents remained high at about 200,000. Despite the phased decline in that number, road traffic accidents caused quite a few deaths each year (about 60,000).

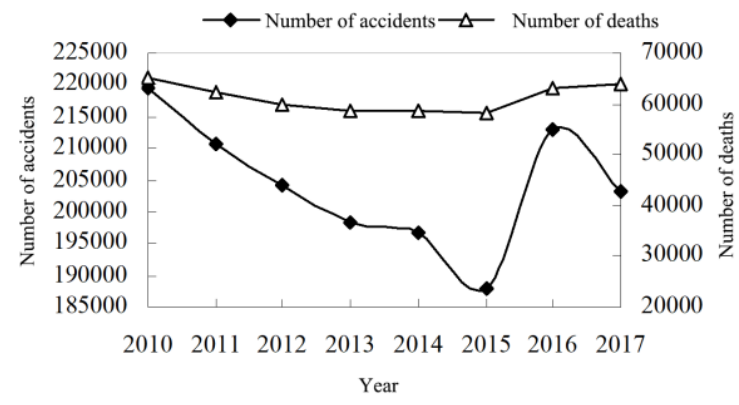

Figure 1. The curves of the number of road traffic accidents and the number of deaths

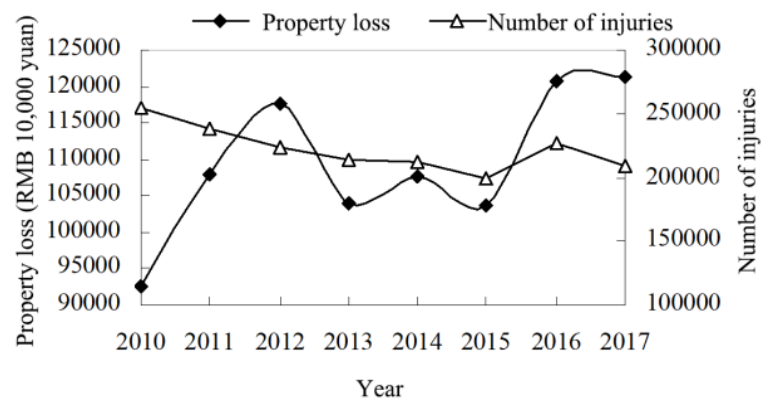

Figure 2. The curves of the number of property loss and the number of injuries

As shown in Figure 2, the property loss induced by road traffic accidents increased with certain fluctuations. The increment was relatively large in several years. For example, the property loss was as high as RMB 1,213,110,000 yuan. The number of injuries caused by road traffic accidents remained high $(>200,000)$, despite a general declining trend. The number of injuries had some fluctuations: the number decreased all the way until 2015, rebounded in 2016, and reduced again in 2017.

\section{GREY PREDICTION OF ROAD TRAFFIC ACCIDENTS}

Proposed by Prof. Deng Julong in 1982, the grey system theory holds that there is an inherent law of any complex phenomenon, because each phenomenon has an overall function. It is very important to select a suitable tool to mine and utilize the law. To disclose the regularity of any grey sequence, the stochasticity must be weakened by a suitable generation mechanism. Data sequences can be commonly generated through accumulated generating operation (AGO), inverse AGO, and weighted AGO.

The grey system theory successfully solves numerous practical problems, bringing obvious social and economic benefits. The core of this system is grey modelling. Through grey relational analysis, this paper looks for the causes of road traffic accidents, identifies the main factors affecting traffic safety, and makes scientific predictions of the main parameters of road traffic accidents.

Suppose the original data sequence has $\mathrm{n}$ statistical indices:

$$
x^{(0)}(t)=\left\{x^{(0)}(1), x^{(0)}(2), \ldots, x^{(0)}(n)\right\}
$$

Perform an AGO on the original data sequence, the generated data sequence $x^{(1)}(t)$ can be obtained as:

$$
x^{(1)}(t)=\left\{x^{(1)}(1), x^{(1)}(2), \ldots, x^{(1)}(n)\right\}
$$

where,

$$
x^{(1)}(t)=\sum_{\mathrm{t}}^{k=1} x^{(0)}(k)
$$

The relationship between the generated data sequence and the original data sequence is as follows: 


$$
x^{(0)}(n)=x^{(1)}(n)-x^{(1)}(n-1)
$$

Then, the GM $(1,1)$ model can be established as:

$$
x^{(0)}(k)+a z^{(1)}(k)=b
$$

The whitenization differential equation can be expressed as:

$$
\frac{d x^{(1)}}{d t}+a X^{(1)}=b
$$

where, $a$ and $b$ are constants. Let $\hat{A}$ be the vector of the parameter to be estimated. By the least squares (LS) method:

$$
\begin{gathered}
\hat{A}=\left[B^{T} B\right]^{-1} B^{T} Y \\
B=\left[\begin{array}{cc}
-\frac{1}{2}\left[x^{(1)}(1)+x^{(1)}(2)\right] & 1 \\
-\frac{1}{2}\left[x^{(1)}(2)+x^{(1)}(3)\right] & 1 \\
\ldots & \ldots \\
-\frac{1}{2}\left[x^{(1)}(n-1)+x^{(1)}(n)\right] & 1
\end{array}\right] \\
Y=\left[x^{(0)}(2), x^{(0)}(3), \ldots, x^{(0)}(n)\right]^{T}
\end{gathered}
$$

Solving the differential equation:

$$
\begin{aligned}
& \hat{x}^{(1)}(k+1)=\left[x^{(0)}(1)-\frac{b}{a}\right] e^{-a k}+\frac{b}{a}, \\
& k=0,1,2, \cdots, n
\end{aligned}
$$

The above formula is the prediction formula of the original data sequence. Through the IAGO, the original data sequence can be recovered as:

$$
\left\{\begin{array}{c}
\hat{x}^{(0)}(1)=x^{(0)}(1) \\
\hat{x}^{(0)}(k)=\hat{x}^{(1)}(k)-\hat{x}^{(1)}(k-1)= \\
{\left[x^{(0)}(1)-\frac{b}{a}\right]\left(1-e^{a}\right) e^{-a(k-1)}, k>1}
\end{array}\right.
$$

The number of road traffic accidents from 2010 to 2017 forms an original discrete data sequence: $x^{(0)}=[219521,210812,204196,198394,196812,187781$, 212846, 203049].

The following data sequence can be obtained through the AGO:

$x^{(1)}=[219521,430333,634529,832923,1029735,1217516$, $1430362,1633411]$

Further, data matrices B and Y can be established as:

$$
B=\left[\begin{array}{cc}
-324927 & 1 \\
-532431 & 1 \\
-733726 & 1 \\
-931329 & 1 \\
-1123625.5 & 1 \\
-1323939 & 1 \\
-1531886.5 & 1
\end{array}\right]
$$

$Y=[210812,204196,198394,196812,187781,212846$, $203049]^{T}$

According to formula (7), we have:

$$
\hat{a}=\left[B^{T} B\right]^{-1} B^{T} Y
$$

Substituting relevant data into the above formula, we have $a=0.0030$ and $b=204,771.1596$.

Substituting the values of $a$ and $b$ into the differential equation, the prediction model can be established as:

$$
\begin{aligned}
& \hat{x}^{(1)}(k+1) \\
& =\left[x^{(0)}(1)-\frac{b}{a}\right] e^{-a k}+\frac{b}{a} \\
& =-68028697.93 e^{-0.0030 k}+68248218.9276
\end{aligned}
$$

Based on formula (13), the predicted data sequence $\hat{x}^{(0)}$ of the original data sequence $x^{(0)}$ can be obtained:

$$
\left\{\begin{array}{l}
\hat{x}^{(0)}(1)=x^{(0)}(1)=219521 \\
\hat{x}^{(0)}(k) \\
=\hat{x}^{(1)}(k)-\hat{x}^{(1)}(k-1)=204419.03 e^{-0.0030(k-1)}, k>1
\end{array}\right.
$$

Table 1. The relative errors between prediction and actual data

\begin{tabular}{ccccc}
\hline Year & Actual value & Predicted value & Absolute error & Relative error \\
\hline 2010 & 219521 & 219521 & 0 & 0 \\
2011 & 210812 & 203806.61 & 7005.39 & 0.0332 \\
2012 & 204196 & 203196.03 & 999.97 & 0.0049 \\
2013 & 198394 & 202587.27 & -4193.27 & 0.0211 \\
2014 & 196812 & 201980.34 & -5168.34 & 0.0263 \\
2015 & 187781 & 201375.23 & -13594.23 & 0.0724 \\
2016 & 212846 & 200771.93 & 12074.07 & 0.0567 \\
2017 & 203049 & 200170.44 & 2878.56 & 0.0142 \\
\hline
\end{tabular}


The predicted data are compared with the actual data in Table 1. It can be seen that the mean relative error of the prediction was $2.86 \%$, indicating that the grey prediction is highly accurate and suitable for medium- and long-term forecasts.

\section{GREY MARKOV MODEL PREDICTION OF ROAD TRAFFIC ACCIDENTS}

The Markov chain can predict the future based on the latest dynamic data. Meanwhile, the causes of road traffic accidents are sudden and stochastic. The situation at the current moment does not affect the probability of road traffic accident at the next moment. Therefore, it is very suitable to predict road traffic accidents by the Markov chain. This paper collects the data on road traffic accidents in 2010-2017, and then predicts such accidents based on grey Markov model.
From the abovementioned GM $(1,1)$ model, we have $a=0.0030$ and $b=204,771.1596$.

$$
\hat{x}^{(0)}(k+1)=\left[x^{(0)}(1)-\frac{b}{a}\right]\left(1-e^{a}\right) e^{-a k}
$$

Thus, we have $\hat{x}^{(0)}(k+1)=68233091.46 e^{-0.0030 k}$.

During prediction, the Markov model judges the state of the object at the next moment according to the current state and the state transition probability matrix. Thus, the prediction takes basis on the division of states: the accuracy of prediction hinges on the appropriateness of state division. Here, the original data sequence is divided based on the actual situation (Table 2).

According to the state division, the number of road traffic accidents in 2010-2017 can be expressed as in Table 3.

Table 2. State division of the number of road traffic accidents

\begin{tabular}{cccc}
\hline States & E1 & E2 & E3 \\
\hline$\frac{x^{(0)}(k)}{\hat{x}^{(0)}(k)}$ & $0.9320 \sim 0.9880$ & $0.9880 \sim 1.0100$ & $1.0100 \sim 1.1000$ \\
\hline
\end{tabular}

Table 3. The number of road traffic accidents in 2010-2017 based on state division

\begin{tabular}{ccccc}
\hline Year & $\mathrm{X}^{(0)}(\mathrm{k})$ & $\widehat{\boldsymbol{x}}^{(\mathbf{0})}(\boldsymbol{k})$ & $\frac{x^{(0)}(k)}{\hat{x}^{(0)}(k)}$ & State \\
\hline 2010 & 219521 & 219521 & 1 & 2 \\
2011 & 210812 & 203806.61 & 1.0344 & 3 \\
2012 & 204196 & 203196.03 & 1.0049 & 2 \\
2013 & 198394 & 202587.27 & 0.9793 & 1 \\
2014 & 196812 & 201980.34 & 0.9744 & 1 \\
2015 & 187781 & 201375.23 & 0.9325 & 1 \\
2016 & 212846 & 200771.93 & 1.0601 & 3 \\
2017 & 203049 & 200170.44 & 1.0144 & 3 \\
\hline
\end{tabular}

The 1-step state transition probability matrix can be derived from the number of road traffic accidents based on state division:

$$
P_{1}=\left[\begin{array}{ccc}
\frac{2}{3} & 0 & \frac{1}{3} \\
\frac{1}{2} & 0 & \frac{1}{2} \\
0 & \frac{1}{2} & \frac{1}{2}
\end{array}\right]
$$

From the 1-step state transition probability matrix, the number of road traffic accidents in 2010-2017 was predicted. The predicted results are recorded in Table 4.

According to the absolute distribution, the number of road traffic accidents in 2018 and 2019 were predicted as 189,521 and 186,344 , respectively.

Table 4. The predicted number of road traffic accidents in 2010-2017

\begin{tabular}{c|cccc}
\hline Year & 2010 & 2011 & 2012 & 2013 \\
Predicted value & 219521 & 210773 & 204643 & 196660 \\
Year & 2014 & 2015 & 2016 & 2017 \\
Predicted value & 197170 & 191915 & 208672 & 207135 \\
\hline
\end{tabular}

\section{COMPARATIVE ANALYSIS}

Both grey prediction and grey Markov model are applicable to the prediction of road traffic accidents.

As shown in Table 5, the absolute errors of grey prediction maximized at 13,594 (relative error: $7.24 \%$ ), and minimized at 1,000 (relative error: $0.49 \%$ ); the absolute errors of grey Markov model maximized at 4,174 (relative error: $1.96 \%$ ), and minimized at 39 (relative error: $0.02 \%$ ). The grey Markov model clearly outshined the grey prediction in accuracy.

Table 5. Comparison between the prediction results of grey prediction and grey Markov model

\begin{tabular}{cccccc}
\hline Year & $\begin{array}{c}\text { Actual } \\
\text { value }\end{array}$ & $\begin{array}{c}\text { Grey } \\
\text { prediction }\end{array}$ & $\begin{array}{c}\text { Absolute } \\
\text { error }\end{array}$ & $\begin{array}{c}\text { Grey } \\
\text { Markov } \\
\text { model }\end{array}$ & $\begin{array}{c}\text { Absolute } \\
\text { error }\end{array}$ \\
\hline 2010 & 219521 & 219521 & 0 & 219521 & 0 \\
2011 & 210812 & 203806.61 & 7005.39 & 210773 & 39 \\
2012 & 204196 & 203196.03 & 999.97 & 204643 & -447 \\
2013 & 198394 & 202587.27 & -4193.27 & 196660 & 1734 \\
2014 & 196812 & 201980.34 & -5168.34 & 197170 & -358 \\
2015 & 187781 & 201375.23 & -13594.23 & 191915 & -4134 \\
2016 & 212846 & 200771.93 & 12074.07 & 208672 & 4174 \\
2017 & 203049 & 200170.44 & 2878.56 & 207135 & -4086 \\
\hline
\end{tabular}




\section{DISCUSSION}

\subsection{Influencing factors of road traffic accidents}

Currently, the public has a weak awareness of traffic safety. Serious traffic violations, e.g. drunk driving, are very common. Road traffic accidents are mainly caused by human factors, vehicle factors, road factors, and environmental factors. These factors are detailed as follows:

(1) Human is the primary factor of traffic safety. Relevant statistics show that $80-90 \%$ of road traffic accidents are caused by human factors. During driving, the driver's state depends heavily on these factors. The prerequisite for traffic safety lies in the effective education and management of drivers.

(2) Vehicles are a key factor in traffic safety. The traffic safety varies with the status and type of vehicles. Motor vehicles have much greater impacts on traffic safety than bicycles and other non-motor vehicles. Motor vehicles are the most powerful objects on roads, and the culprit of many road traffic accidents.

(3) Roads are also very important to traffic safety. However, there is a huge regional difference in road conditions. In many regions, the roads cannot satisfy the demand of modern transport. There are two possible reasons: the road network in many cities was formed in the era of horse-drawn carriages; the traffic flow of vehicles changes very rapidly.

(4) The traffic environment is a comprehensive factor of traffic safety. The traffic environment takes effect throughout the driving process, exerting a certain impact on the driver's state. However, many drivers have not recognized the importance of the traffic environment. The neglection brings about many accidents that could have been avoided, resulting in huge injuries.

\subsection{Countermeasures for road traffic accidents}

Based on the features and predictions of road traffic accidents, the following countermeasures were put forward to reduce the occurrence of such accidents:

(1) The government should establish a special department to formulate work plans and relevant policies on traffic safety for different periods, and mine out useful information through thorough analysis of the available data. The relevant mechanisms should be created and improved to protect traffic safety.

(2) The social forces should be fully mobilized, such that all traffic participants join hands to ensure traffic safety and mange road traffic accidents in a comprehensive manner. Every party should consider the safety of others, in addition to that of their own. The penalties could be increased to promote the awareness of traffic safety among traffic participants.

(3) Individuals should pay sufficient attention to traffic safety. The violations by traffic participants are the leading cause of road traffic accidents. Even if there are strict regulations and sufficient supports, road traffic accidents will occur, as long as traffic participants fail to recognize the importance of traffic safety. Therefore, regular publicity must be implemented to educate individuals which unsafe behaviours will lead to road traffic accidents, and what are the serious consequences of such accidents. Individuals must pay high attention to traffic safety and strictly adhere to the relevant regulations.

\section{CONCLUSIONS}

This paper statistically analyses the features of road traffic accidents, and predicts such accidents by grey prediction and grey Markov model. Based on the predicted results, the authors analysed the causes and influencing factors of such accidents, and put forward several countermeasures for traffic safety.

(1) The authors analysed the features of road traffic accidents in China in recent years, including the number of accidents, the number of deaths, the number of injuries, and property loss. The analysis shows that only the number of injuries was declining, while the trends of the other three factors are not optimistic.

(2) The number of road traffic accidents was predicted separately based on the grey system theory and the grey Markov model. Error analysis shows that the two prediction methods both apply to the prediction of road traffic accidents, but differ in prediction accuracy.

(3) Contrastive analysis shows that grey prediction had a maximum error of 13,594 and a minimum error of 1,000, while grey Markov model had a maximum error of 5,984 and a minimum error of 478. The prediction accuracy of grey Markov model is clearly better than that of grey prediction.

(4) Road traffic accidents are mainly affected by human factors, vehicle factors, road factors, and environmental factors. After analysing these factors, several countermeasures were designed, calling the government, the society, and individuals to work together to prevent road traffic accidents.

\section{REFERENCES}

[1] Eboli, L., Forciniti, C., Mazzulla, G. (2020). Factors influencing accident severity: An analysis by road accident type. Transportation Research Procedia, 47: 449-456. https://doi.org/10.1016/j.trpro.2020.03.120

[2] Barbone, F., McMahon, A.D., Davey, P.G., Morris, A.D. Reid, I.C., McDevitt, D.G., MacDonald, T.M. (1998). Association of road-traffic accidents with benzodiazepine use. The Lancet, 352(9137): 1331-1336. https://doi.org/10.1016/S0140-6736(98)04087-2

[3] Elvik, R., Christensen, P., Amundsen, A. (2004). Speed and road accidents. An evaluation of the Power Model. TØI Report, 740: 2004.

[4] Edwards, J.B. (1998). The relationship between road accident severity and recorded weather. Journal of Safety Research, 29(4): 249-262. https://doi.org/10.1016/S0022-4375(98)00051-6

[5] Aarts, L., Van Schagen, I. (2006). Driving speed and the risk of road crashes: A review. Accident Analysis \& Prevention, 38(2) https://doi.org/10.1016/j.aap.2005.07.004

[6] Anderson, T.K. (2009). Kernel density estimation and Kmeans clustering to profile road accident hotspots. Accident Analysis \& Prevention, 41(3): 359-364. https://doi.org/10.1016/j.aap.2008.12.014

[7] Deng, J.L. (2002). Grey Theory Basis. Huazhong University of Science and Technology Press.

[8] Xu, G.X., Li, X.S., Lin, Q.F., Wang, Q. (2005). Gray model $\operatorname{GM}(1,1)$ for forecast of traffic accidents. Journal of Shandong University of science and Technology (Natural Science Edition), 19(6): 45-49. https://doi.org/10.3969/j.issn.1672-6197.2005.06.011 
[9] Liu, Z.H., Wang, C., Xu, H.G. (2006). Grey prediction model for road traffic accident and its application research. Journal of Changchun University of Science and Technology (Natural Science Edition), 29(4): 42-44. https://doi.org/10.3969/j.issn.1672-9870.2006.04.013

[10] Mao, Z.L., Sun, J.H. (2011). Application of GreyMarkov model in forecasting fire accidents. Procedia Engineering, 11: 314-318. https://doi.org/10.1016/j.proeng.2011.04.663

[11] Kumar, U., Jain, V. K. (2010). Time series models (GreyMarkov, Grey Model with rolling mechanism and singular spectrum analysis) to forecast energy consumption in India. Energy, 35(4): 1709-1716. https://doi.org/10.1016/j.energy.2009.12.021

[12] Wu, J.H., Lauh, C.R. (1998). A study to improve GM (1, 1) via heuristic method. The Journal of Grey System, 10(3): 183-192.

[13] Chen, K.M., Xie, L.F., Xiang, W.S. (2012). Traffic accidents prediction using improved Grey-Markov model. In Advanced Materials Research, 378: 222-225. https://doi.org/10.4028/www.scientific.net/AMR.378379.222

[14] He, Y., Bao, Y.D. (1992). Grey-Markov forecasting model and its application. System Engineering-Theory \& Practice, 9(4): 59-63.

[15] Wang, P., Lv, Y.B. (2010). Research of traffic accident prediction method. Agriculture Network Information, 6: 130-133. https://doi.org/10.3969/j.issn.16726251.2010 .06 .043

[16] Li, Q.F., Hu, Q.F., Zhang, P. (2007). Application of Grey-Markov model in predicting traffic volume. 2007 IEEE International Conference on Grey Systems and Intelligent Services, Nanjing, China, pp. 707-711. https://doi.org/10.1109/GSIS.2007.4443366

[17] Li, X.Y., Zhang, N., Jiang, G.F. (2003). Grey-Markov model for forecasting road accidents. Journal of Highway and Transportation Research and Development, 20(4): 98-100, 104. https://doi.org10.3969/j.issn.10020268.2003.04.027

[18] Hu, S. (2017). Prediction of city traffic accidents based on grey-Markov chain model. Jurnal Revista de la Faculated de Ingeniea UCV, 32(4): 144-151.

[19] Wang, G. (2006). Grey-Markov model for forecasting road accidents. Journal of Guangdong Communications Polytechnic, $5(1)$ : 30-32. https://doi.org/10.3969/j.issn.1671-8496.2006.01.009

[20] Lin, Y., Chen, S., Chen, Y., Zhai, J. (2013). GreyMarkov model and algorithm for traffic accidents forecasting. Journal of Wuhan University of Technology (Transportation Science \& Engineering), 37(5): 924-929. https://doi.org/10.3963/j.issn.2095-3844.2013.05.007

[21] Zhao, J.N., Wu, Z.L. (2005). Forecasting of maritime accidents by grey-Markov model. Journal of Dalian Maritime University, 4: 15-17.
[22] Lee, S., Lee, T., Kim, H. J., Lee, Y. (2005). Development of traffic accidents prediction model with intelligent system theory. In International Conference on Computational Science and Its Applications, Springer, Berlin, Heidelberg, 3481: 880-888. https://doi.org/10.1007/11424826_95

[23] Liu, L.B., Pei, Y., Pei, T.S. (2019). Application of Grey Markov model in bridge operation prediction. Journal of Hebei University (Natural Science Edition), 39(1): 11-17. http://xbzrb.hbu.cn/EN/10.3969/j.issn.10001565.2019.01.003

[24] Chen, D.Q., Zhu, D.L. (2013). Analysis and prediction of seasonal variation rules of highway traffic accidents. Journal of Safety Science and Technology, 9(3): 188-192. https://doi.org/10.11731/j.issn.1673-193x.2013.03.34

[25] Deng, J.L. (1992). Course of Grey System Theory. Wuhan: Huazhong University of Technology Press.

[26] Liu, S.F. (2014). Grey System Theory and Its Application. Science Press.

[27] Sun, X., Sun, W., Wang, J., Zhang, Y., Gao, Y. (2016). Using a Grey-Markov model optimized by Cuckoo search algorithm to forecast the annual foreign tourist arrivals to China. Tourism Management, 52: 369-379. https://doi.org/10.1016/j.tourman.2015.07.005

[28] Samet, H., Mojallal, A. (2014). Enhancement of electric arc furnace reactive power compensation using GreyMarkov prediction method. IET Generation, Transmission \& Distribution, 8(9): 1626-1636. https://doi.org/10.1049/iet-gtd.2013.0698

[29] Gasparini, M. (1997). Markov chain monte carlo in practice. Technometrics, 39(3): 338-338. https://doi.org/10.1080/00401706.1997.10485132

[30] Ronquist, F., Teslenko, M., Van Der Mark, P., Ayres, D.L., Darling, A., Höhna, S., Larget, B., Liu, L., Suchard, M.A., Huelsenbeck, J.P., Huelsenbeck, J.P. (2012). MrBayes 3.2: Efficient Bayesian phylogenetic inference and model choice across a large model space. Systematic Biology, 61(3): 539-542. https://doi.org/10.1093/sysbio/sys029

[31] Pham, C., Tran, N.H., Ren, S., Saad, W., Hong, C.S. (2017). Traffic-aware and energy-efficient vNF placement for service chaining: Joint sampling and matching approach. IEEE Transactions on Services Computing, 13(1): 172-185. https://doi.org/10.1109/TSC.2017.2671867

[32] Green, P.J. (1995). Reversible jump Markov chain Monte Carlo computation and Bayesian model determination. Biometrika, 82(4): 711-732. https://doi.org/10.1093/biomet/82.4.711

[33] Rabiner, L., Juang, B. (1986). An introduction to hidden Markov models. IEEE ASSP Magazine, 3(1): 4-16. https://doi.org/10.1109/MASSP.1986.1165342 